\title{
Glossopharyngeal Nerve Regeneration Is Essential for the Complete Recovery of Quinine-Stimulated Oromotor Rejection Behaviors and Central Patterns of Neuronal Activity in the Nucleus of the Solitary Tract in the Rat
}

\author{
Camille T. King, ${ }^{1}$ Mircea Garcea, ${ }^{2}$ and Alan C. Spector ${ }^{2}$ \\ ${ }^{1}$ Department of Psychology, Stetson University, DeLand, Florida 32720, and 2Department of Psychology, University of \\ Florida, Gainesville, Florida 32611
}

\begin{abstract}
The peripheral, central, and behavioral consequences of glossopharyngeal nerve transection (GLX), regeneration, and the prevention of regeneration on the quinine-elicited responses of adult rats were concurrently examined. Oromotor taste reactivity (TR) was videotaped during intraoral infusion of $7 \mathrm{ml}$ of either quinine (3 $\mathrm{mm}$ ) or distilled water at 17,52 , or $94 \mathrm{~d}$ after surgery. We confirmed previous findings by showing that $17 \mathrm{~d}$ after neurotomy, (1) the number of circumvallate (CV) and foliate taste buds, (2) gapes (a characteristic aversive TR response), and (3) the number of Fos-like immunoreactive (FLI) neurons in the gustatory NST (gNST), particularly in the medial portion (subfield 5) of the rostral central subdivision (RC), were all severely attenuated in GLX rats. We extended these findings by showing that these lesion-induced effects were enduring when the GL did not regenerate (up to $94 \mathrm{~d}$ ). In contrast, when the GL regenerated, as few
\end{abstract}

Although it has been known for some time that the lingual gustatory nerves have a great proclivity to regenerate after transection, the functional consequences of the loss and recovery of nerve input have only begun to be examined. In the somatosensory system, nerve damage and subsequent regeneration lead to striking reorganizational events that are manifest in both the morphological and physiological properties of neurons in central structures (Jain et al., 1998; Kis et al., 1999). Whether the gustatory system has the potential to express similar neural plasticity remains primarily unexplored, with a few notable exceptions (Whitehead et al., 1995; Barry, 1999). Toward this end, we demonstrated recently that transection of the glossopharyngeal nerve (GL), which innervates taste buds on the posterior tongue, changes the characteristic magnitude and pattern of quinine-induced Fos-like immunoreactivity (FLI) in the rostral nucleus of the solitary tract (rNST) (King et al., 1999), the first central relay in the gustatory system. Intraoral infusion of quinine, an alkaloid that tastes bitter to humans, activates a topographically distinct population of cells in the dorsomedial portion of the rNST as indexed by FLI (Harrer and Travers, 1996). Bilateral transection of the GL (GLX) reduces the number of FLI-positive neurons in the rNST activated by quinine and

\footnotetext{
Received May 8, 2000; revised Aug. 16,. 2000; accepted Aug. 18, 2000.

This work was supported in part by Grant R01-DC01628 (A.C.S.) from the National Institute on Deafness and Other Communication Disorders. A.C.S. is the recipient of Research Career Development Award K04-DC00104 from the National Institute on Deafness and Other Communication Disorders. Parts of this paper were presented at the 21st Meeting of the Association for Chemoreception Sciences in Sarasota, FL, April, 1999. We extend our appreciation to Dr. Michael S. King and the Stetson University Biology Department for the generous use of their microscope, image analysis system, and video equipment. We also thank Suzanne Sealey, Kim Robertson, and Sonia Bretzmann for technical assistance.

Correspondence should be addressed to Dr. Alan C. Spector, Department of Psychology, University of Florida, Gainesville, FL 32611-2250. E-mail: spector@ psych.ufl.edu.

Copyright (C) 2000 Society for Neuroscience $0270-6474 / 00 / 208426-09 \$ 15.00 / 0$
}

as $52 \mathrm{~d}$ were sufficient to re-establish quinine-elicited TR, especially gaping, and FLI expression in RC, particularly within subfield 5 , to values comparable with quinine-stimulated shamoperated rats. Evidently, the gNST maintains its potential to restore accurately the organization of neural activity that is disrupted by nerve injury, as assessed by FLI, ultimately leading to the return of normal protective oromotor responses, provided the nerve regenerates. This recovery was complete despite the reappearance of a reduced population of CV taste buds ( $\sim 75 \%$ control values) and may relate to peripheral and/or central changes that occur in tandem with regeneration of the GL.

Key words: taste; nerve transection; regeneration; Fos immunohistochemistry; functional recovery; taste reactivity; glossopharyngeal nerve; bitter

changes the spatial distribution of the labeled neurons such that these features are indistinguishable from those after water stimulation (King et al., 1999).

This robust effect is surprising considering that GLX alone is inconsequential to performance on a variety of taste-related tasks involving quinine. For example, quinine detection thresholds and performance on a quinine versus $\mathrm{KCl}$ discrimination task are both unaffected by bilateral transection of the nerve (St. John and Spector, 1996, 1998). On the other hand, GLX is not entirely without effect on behavioral responses to quinine in rats (Travers et al., 1987; Grill and Schwartz, 1992; Grill et al., 1992; St. John and Spector, 1998; Markison et al., 1999). Most notably, GLX causes a marked reduction in the number of gapes, a stereotypical oromotor response to orally delivered aversive chemical compounds, elicited by quinine (Travers et al., 1987; Grill et al., 1992). Collectively, these findings suggest that the rNST neurons expressing FLI in response to quinine stimulation of taste receptors may be preferentially involved in the neural circuit controlling protective oromotor rejection reflexes and that the quinine signals transmitted in the GL play a primary role in triggering the response.

Regardless of one's theoretical orientation on the meaning of these results, the fact remains that GLX in the rat leads to measurably robust and reliable changes in neuronal activation patterns in the rNST, as well as attenuated oromotor rejection behaviors in response to intraorally delivered quinine. As such, this paradigm represents a useful model for studying the possibility that the change in the stimulus-induced topography of FLI provoked by nerve transection undergoes further alterations as a function of either time or nerve regeneration. This same point applies to the behavior as well. Accordingly, we hypothesized that regeneration of the GL after its transection would lead to the behavioral recovery of the gape response and restore the characteristic pattern of FLI activation in the rNST associated with quinine stimulation of oral sensory receptors. 
Table 1. Summary of the number of subjects assigned to each experimental group and stimulus condition

\begin{tabular}{|c|c|c|c|c|}
\hline \multirow[b]{2}{*}{ Nerve surgery } & \multirow{2}{*}{$\begin{array}{l}\text { Number of days } \\
\text { before test day }\end{array}$} & \multicolumn{2}{|c|}{$\begin{array}{l}\text { Number of subjects } \\
\text { per test day stimulus }\end{array}$} & \multirow{2}{*}{$\begin{array}{l}\text { Abbreviation } \\
\text { in text }\end{array}$} \\
\hline & & (Quinine) & (Water) & \\
\hline GL exposed & 17 & 6 & 6 & SHAM-17 \\
\hline GL transected & 17 & 6 & 6 & GLX \\
\hline GL transected & 52 & 6 & 5 & REG-52 \\
\hline GL excised & 52 & 6 & 5 & PRE-52 \\
\hline GL exposed & 94 & 7 & 6 & SHAM-94 \\
\hline GL transected & 94 & 6 & 6 & REG-94 \\
\hline GL excised & 94 & $6(3)^{*}$ & $6(5)^{*}$ & PRE-94 \\
\hline
\end{tabular}

*Because of the presence of numerous TBs in the CV and FOL, four subjects in the PRE-94 group were removed from data analyses (see Results for details). The revised $n$ values are in parentheses.

\section{MATERIALS AND METHODS}

Subjects. Eighty-three male Sprague Dawley rats (Charles River Laboratories, Wilmington, MA) weighing $250-275 \mathrm{gm}$ at the time of nerve surgery were individually housed in hanging wire mesh cages where light cycle (lights from 6:00 A.M. to 6:00 P.M.), temperature, and humidity were automatically controlled. All manipulations were performed during the light phase. Laboratory chow (5001; Purina Mills Inc., St. Louis, MO) and water were available ad libitum.

Surgical procedures. Table 1 summarizes the experimental groups. Rats were assigned to one of three surgical conditions: one in which the GL was transected bilaterally $(n=35)$, a second in which $8-10 \mathrm{~mm}$ of the GL was excised bilaterally $(n=23)$, and a control condition in which the GL nerves were simply exposed $(n=25)$. It was anticipated that the GL would not regenerate in $17 \mathrm{~d}$ (GLX) (King et al., 1999) but that 52 or $94 \mathrm{~d}$ would be sufficient for some regeneration to occur (REG groups) (Guth, 1957; S. J. St. John and A. C. Spector, unpublished observations). Moreover, it was expected that the removal of a large portion of the nerves would prevent successful regeneration (PRE groups).

Bilateral glossopharyngeal nerve surgeries were performed 17,52 , or $94 \mathrm{~d}$ before behavioral testing (Table 1). For details of the surgical procedures, refer to St. John et al. (1994). Intraoral cannulas, through which taste stimuli could be directly infused into the oral cavity (Grill and Norgren, 1978; King et al., 1999), were implanted bilaterally 2 weeks before the commencement of behavioral procedures for each rat. For $3 \mathrm{~d}$ after implantation of the cannulas, all rats received subcutaneous injections of penicillin $(30,000 \mathrm{U})$. Additional injections were given as needed throughout the recovery period to any animal showing signs of infection. Wet mash (powdered chow mixed with water and supplemented with a calorically dense suspension (Nutri-Cal; Evsco Pharmaceuticals, Buena, $\mathrm{NJ}$ ) was available ad libitum to all rats for $3-5 \mathrm{~d}$ to promote feeding. The cannulas were cleaned daily to maintain patency and prevent infection. Animals recovered from the intraoral cannula surgery for $14 \mathrm{~d}$, at which time behavioral procedures began.

Stimulus delivery. Behavioral procedures were based on those described previously (King et al., 1999). On each of the $3 \mathrm{~d}$ before the test day (see below), subjects were familiarized with the behavioral arena and infusion process. The procedures on habituation days were followed exactly as described below, except that all animals received distilled water $\left(\mathrm{dH}_{2} \mathrm{O}\right)$ as the intraoral stimulus. On the test day, which occurred 17, 52, or $94 \mathrm{~d}$ after nerve surgery, the animal's left cannula was attached via polyethylene tubing to a syringe on an infusion pump (Harvard Apparatus, South Natick, MA), and the animal was placed in a cylindrical Plexiglas chamber for a $1 \mathrm{hr}$ adaptation period. For the next $30 \mathrm{~min}, 7 \mathrm{ml}$ of either $\mathrm{dH}_{2} \mathrm{O}$ or $3 \mathrm{~mm}$ quinine-hydrochloride was infused through the cannula at a rate of $0.233 \mathrm{ml} / \mathrm{min}$. During the $1 \mathrm{st}$ and 30th minutes, an experimenter was present in the room to video tape the rat for subsequent scoring of taste reactivity (TR) behaviors. Responses were recorded at 30 frames/sec using a video camera $(5100 \mathrm{HS}$; Panasonic, Secaucus, NJ) and recorder (model SR-S365U; JVC, Wayne, NJ). At all other times, no experimenter was present in the room. At the end of the 30th minute, the infusion pump was turned off. The animal remained in the chamber for $45 \mathrm{~min}$ before either being returned to its home cage (habituation days) or anesthetized for perfusion (test day).

Brain and tongue histology. Immediately after the $45 \mathrm{~min}$ postinfusion period on the test day, animals were deeply anesthetized with an overdose of sodium pentobarbital and perfused intracardially with heparinized 0.15 $\mathrm{M} \mathrm{NaCl}$, followed by sodium phosphate buffered $4 \%$ paraformaldehyde, $\mathrm{pH}$ 7.3. Brains were removed and post-fixed overnight at $4^{\circ} \mathrm{C}$. Each brain was then cut in the coronal plane $(75 \mu \mathrm{m})$ using a vibratome. Every other section was processed for Fos immunoreactivity. After a 20 min pretreatment with sodium borohydride [1\% in potassium PBS (KPBS)], these sections were rinsed in KPBS and then incubated with rabbit polyclonal antibody [c-Fos (4), sc-52; Santa Cruz Biotechnology, Santa Cruz, CA] at a dilution of $1: 10,000$ in $0.4 \%$ Triton $\mathrm{X}-100$ in KPBS for $72 \mathrm{hr}$ at $4^{\circ} \mathrm{C}$. After several rinses in KPBS, the sections were placed in biotinylated goat anti-rabbit IgG (Zymed, San Francisco, CA) at a dilution of 1:600 for $4 \mathrm{hr}$ at room temperature. They were then rinsed with KPBS before being placed in sodium phosphate buffer containing $0.03 \%$ diaminobenzidine, $0.008 \%$ nickel ammonium sulfate, and $0.0075 \%$ hydrogen peroxide. Finally, the stained sections were mounted on chrome-alum subbed slides, dehydrated, and coverslipped. The alternate sections, used to delineate the borders of the NST and its subdivisions, were mounted, stained with $0.1 \%$ thionin, dehydrated, and coverslipped.

The subjects' tongues were post-fixed in $10 \%$ buffered formalin for several weeks. The portion of the posterior tongue containing the circumvallate papilla $(\mathrm{CV})$ was embedded in paraffin, sectioned at $10 \mu \mathrm{m}$ on a rotary microtome, mounted, and stained with hematoxylin and eosin. The foliate papillae (FOL) on the lateral aspects of the tongue were flattened after the underlying muscle and connective tissue were removed, sectioned, and stained as described above for the CV papilla.

Microscopic analysis of brain and tongue tissues. The tracing and counting of FLI-positive cells in the NST was performed by an experimenter who was unaware of either the surgical group or stimulus condition to which the subjects were assigned. Brain sections were observed under $2-40 \times$ objectives with a Zeiss (Oberkochen, Germany) Axioscope microscope equipped with a video camera coupled to a video monitor and computer. Video images were captured using Zeiss Image software.

Five standard sections of the left NST spaced throughout its rostrocaudal extent were selected for analysis based on the terminal fields of the gustatory nerves (Hamilton and Norgren, 1984). Four of these sections were selected from the gustatory zone of the NST (gNST) rostral to the area postrema (AP). The most rostral of these gustatory sections (RgNST) was taken at the caudal level of the dorsal cochlear nucleus. The most caudal of the gustatory sections (CgNST) was taken $\sim 300 \mu \mathrm{m}$ caudal to where the NST first abuts the fourth ventricle. The two intermediate sections, intermediate rostral (IRgNST) and intermediate caudal (ICgNST) were taken at approximately equidistant points between RgNST and CgNST (for details, see King et al., 1999). One final section was taken at the level of AP and was considered to represent the nongustatory NST. All FLI-positive nuclei within the borders of the NST in each section were identified and tagged for later counting.

Objective quantification of the spatial distribution of FLI neurons within each coronal section was achieved in two manners. First, thionin-stained sections were used to delineate anatomically defined subdivisions of the rNST: rostral lateral (RL), rostral central (RC), ventral (V), and medial (M), as described previously by Whitehead (1988) in the hamster and Halsell et al. (1996) in the rat. These particular subdivisions applied only to the three most rostral sections of the gNST in this study (RgNST, IRgNST, and ICgNST) and therefore, only these three levels were delineated in this manner. These levels-subdivisions are compatible with those used for analysis in previous studies (Harrer and Travers, 1996; King et al., 1999). The traced subdivisions were overlaid onto the Fos images so that the number of FLI-positive cells in each of these subdivisions could be tallied. The second method involved parceling the gNST into six "subfields" based on its mediolateral and dorsoventral dimensions (for details, see King et al., 1999) and counting the number of FLI-positive neurons in each subfield.

The counting of $\mathrm{CV}$ and FOL taste buds was also performed by an experimenter naive to the experimental assignment of the subjects. Under light microscopy, the taste pores within the $\mathrm{CV}$ of every subject, except one SHAM-14 animal, were counted. Taste pores were counted to minimize the possibility of a given taste bud being counted more than once. For the FOL however, not all tissues were available. There were 2 SHAM-14, 2 GLX, 10 REG-52, 8 PRE-52, 10 SHAM-94, 9 REG-94, and 9 PRE-94 rats from which FOLs were obtained.

Behavioral analysis. One experimenter, unaware of the surgical or experimental condition of the subjects, viewed in slow motion and/or frameby-frame the videotaped responses for the first minute of the infusion period. A variety of oromotor TR behaviors, including both aversive and ingestive domains, were scored (Spector et al., 1988). The aversive behaviors scored included gapes, chin rubs, head shakes (HS), and forelimb flails (FF). The ingestive behaviors scored included tongue protrusions, lateral tongue protrusions, mouth movements, paw licks, and paw lick time [defined as the duration of time (in seconds) the animal was licking its paws when discrete occurrences of the behavior were obscured]. The number of occurrences of each behavior during the 1 min interval constituted a score with the exception of paw lick time. Because paw licks occur at a rate of approximately six licks per second (Grill and Norgren, 1978; Spector et al., 1988), paw lick duration scores were multiplied by 6 . These adjusted scores were added to the number of paw licks for a more accurate indication of total paw licking. Total aversive and ingestive scores were compiled by summing the occurrences of all ingestive or aversive behaviors, respectively.

Statistical procedures. The data were first analyzed with higher order multifactor ANOVAs to test for significant main effects and interactions before proceeding with finer detailed analyses. When justified, separate one-way ANOVAs, one for each dependent variable, were conducted using the 14 groups. Only if the ANOVA revealed significant differences were post hoc Fisher's least significant difference tests performed. For clarity in the presentation of the data, only statistically significant comparisons 

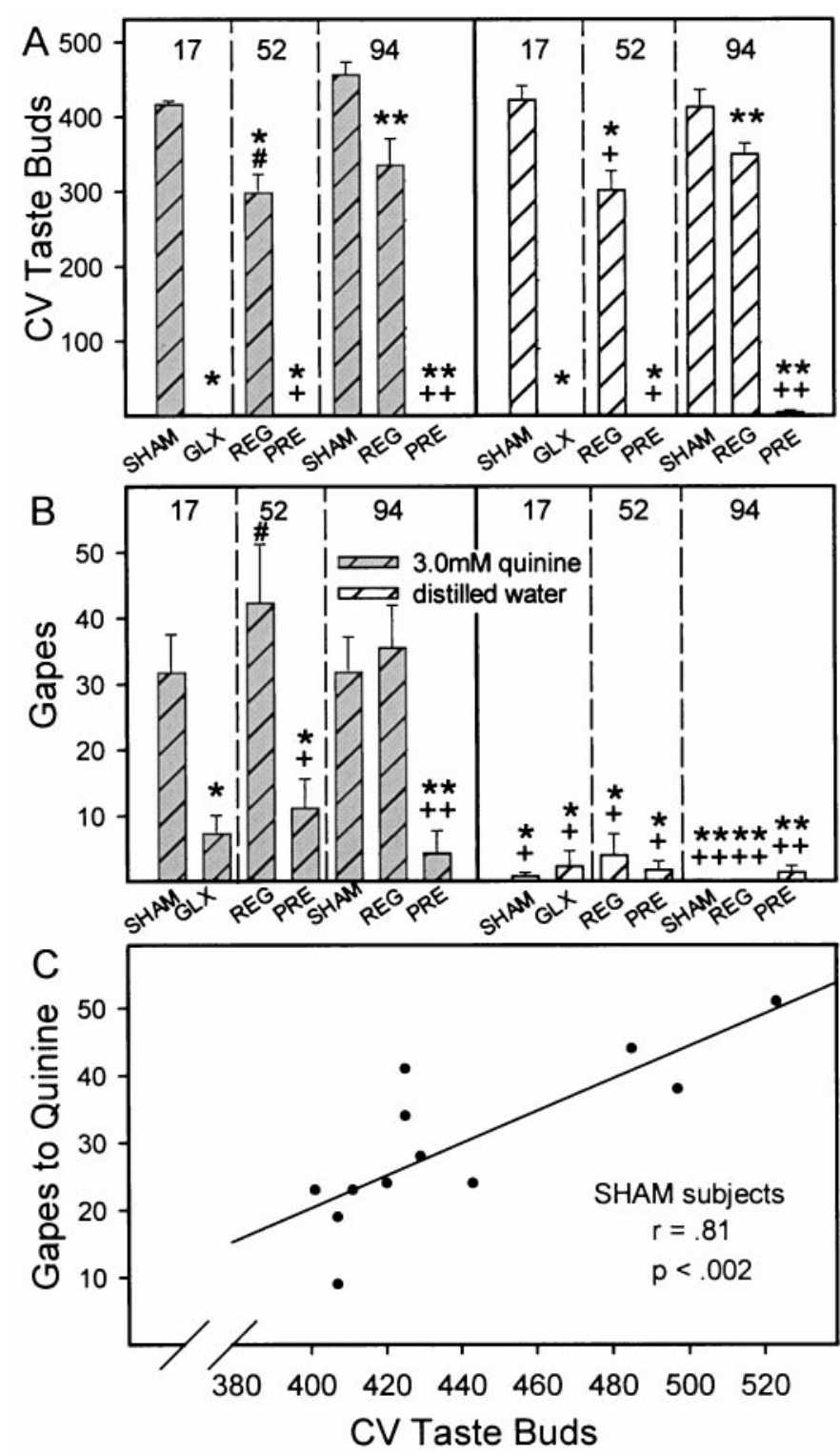

Figure 1. Mean \pm SE numbers of CV taste buds $(A)\left(F_{(13,63)}=116.49 ; p<\right.$ $0.005)$ and gapes $(B)\left(F_{(13,65)}=12.56 ; p<0.005\right)$ in response to intraoral stimulation with $3 \mathrm{~mm}$ quinine or distilled water. In $A$, symbols represent significant differences (at least $p<0.001$ ) within the same-stimulus groups: * indicates a difference from SHAM-17 rats; \# indicates a difference from GLX rats; + indicates difference from REG-52 subjects. Within the $94 \mathrm{~d}$ groups, ${ }^{* *}$ signifies a difference from SHAM-94 rats and ++ indicates a difference from REG-94 subjects. For $B$, and all other figures, the same symbols signify differences from only the quinine-stimulated surgical treatment groups noted above. Notice that, in the absence of an intact CV field (GLX and PRE groups), quinine-elicited gapes were severely attenuated. Regeneration of the GL for at least $52 \mathrm{~d}$ re-established the number of quinine-stimulated gapes to control values. $C$, There was a significant relationship found between the number of CV taste buds and gapes elicited by quinine-stimulation in SHAM rats (both 17 and 94 groups combined).

between the 17 and $52 \mathrm{~d}$ groups and within the SHAM-94 groups are depicted. The "control" condition for both 17 and 52 d groups was the SHAM-17 group, and for the 94 d groups, the SHAM-94 group served this purpose. The statistical rejection criterion used for significance was $p \leq 0.05$.

\section{RESULTS}

\section{Peripheral anatomical consequences of GL transection and regeneration}

\section{Circumvallate papilla}

The absence or presence of CV taste buds in experimental rats (Fig. 1A) was used to ascertain successful nerve transections and the extent of GL regeneration (Guth, 1957; Ganchrow and Ganchrow, 1989). No taste buds were found in the CV of GLX rats, indicating the effectiveness of the nerve surgeries. On the contrary, numerous taste buds were found in rats in which regeneration was encouraged. The abundance of taste buds $(\sim 75 \%$ control values) was interpreted as successful regeneration of the nerve, but it is important to note that as many as $94 \mathrm{~d}$ were not sufficient to restore the typical complement of CV taste buds. Numbers of regenerated CV taste buds, although significantly greater than nerve-transected values (all $p$ values $<0.001$ ), were still significantly less than sham-operated control values at both 52 and $94 \mathrm{~d}$ (all $p$ values $<0.001$ ). Regeneration was successfully prevented in every case in the PRE-52 groups and, in most cases, in the PRE-94 group as indexed by the paucity of CV taste buds. There were four subjects in the PRE-94 group (not represented in Fig. 1) for which the numbers of $\mathrm{CV}$ taste buds were substantial, nearly half of the amount found in SHAM-94 subjects. This was interpreted as regeneration despite our efforts to discourage it, and accordingly, these animals were eliminated from formal data analyses, decreasing by three the number of subjects in the PRE-94 group receiving quinine $(n=3)$ and by one the number receiving water $(n=5)$ (Table 1$)$.

\section{Foliate papillae}

In the GLX (42.50 \pm 2.50$)$, PRE-52 (34.00 \pm 4.46), and PRE-94 $(41.00 \pm 4.68)$ groups, significant reductions in FOL taste bud number were found compared with controls (SHAM-17, $393.00 \pm$ 22.00; SHAM-94, $377.67 \pm 24.07$; all $p$ values $<0.01$ ), but their numbers were never zero. Importantly, the taste buds that persisted in GLX and PRE groups were always located in the most anterior trenches of the FOL, suggesting that they were supported by the
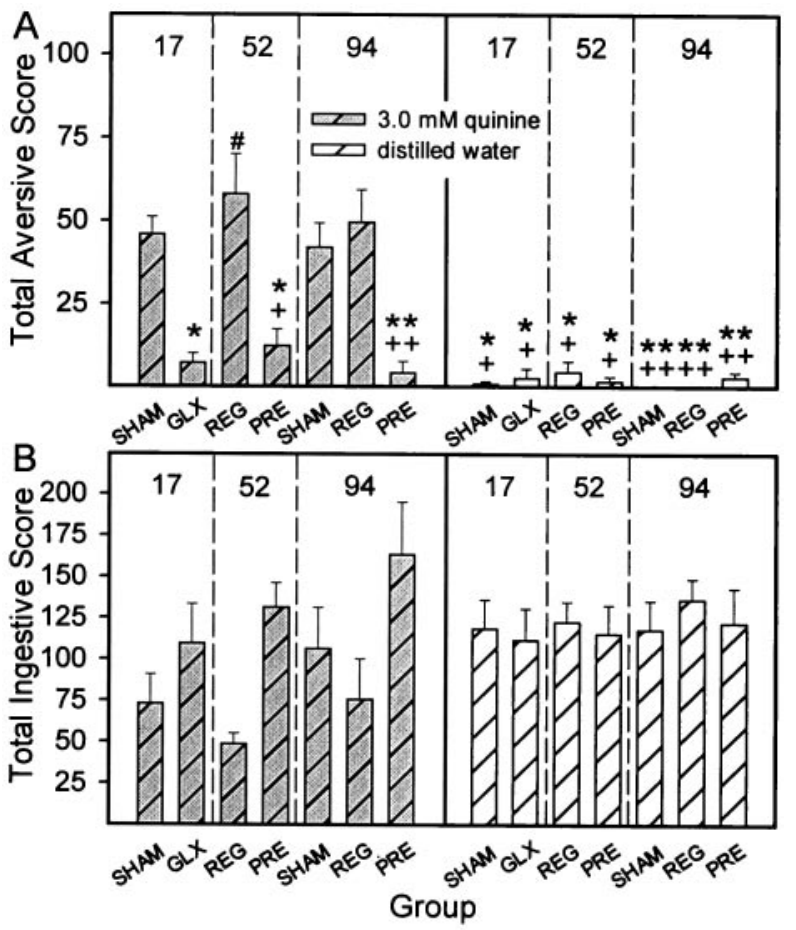

Figure 2. Mean \pm SE total aversive $(A)\left(F_{(13,65)}=15.54 ; p<0.005\right)$ and ingestive $(B)$ scores to the infusion of quinine or water during a $1 \mathrm{~min}$ interval. See Figure 1 for explanation of symbols. A, Bilateral GLX severely reduced the total number of aversive behaviors obtained with quinine stimulation to values obtained when water was the stimulus. This effect was enduring if transection of the GL was permanent as evidenced by the few gapes elicited in the PRE-94 group. On the contrary, concurrent with regeneration of the GL, the numbers of aversive behaviors were comparable with quinine-stimulated SHAM animals. $B$, Ingestive behaviors to quinine stimulation tended to increase in rats with permanent GL nerve transection. In the groups lacking regenerated GLs, the numbers of quinine-stimulated behaviors closely resembled those obtained when water was the stimulus. 
Table 2. Occurrence of individual aversive behaviors

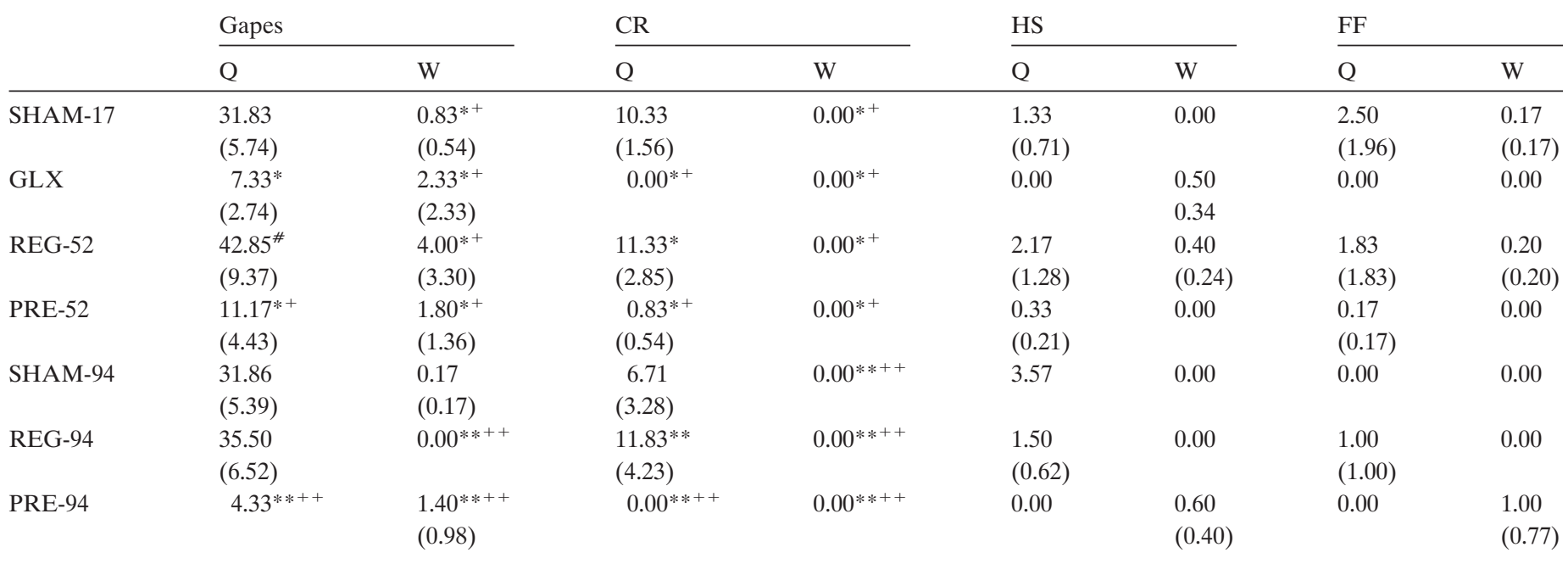

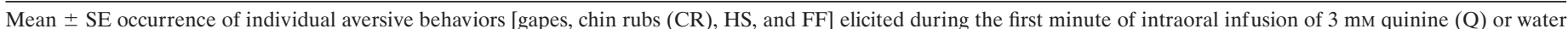

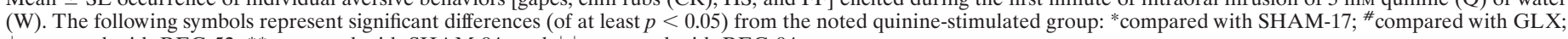
${ }^{+}$compared with REG-52; ** compared with SHAM-94; and ${ }^{++}$compared with REG-94.

chorda tympani nerve (CT), rather than GL, nerve fibers (Miller, 1977). After $52(250.90 \pm 20.60)$ or $94(286.40 \pm 14.89) \mathrm{d}$ of regeneration, the numbers of FOL taste buds were $\sim 64 \%$ of SHAM-17 or $76 \%$ of SHAM-94 values, respectively. Although these numbers represent robust regeneration of the GL, they still remain significantly reduced compared with the undisturbed populations of FOL taste buds (all $p$ values $<0.01$ ). The same four PRE-94 rats showing nearly $50 \%$ of the control number of taste buds in the CV (see above) also had nearly $50 \%$ of their FOL taste buds, providing further evidence for partial regeneration of the GL in these animals supporting their removal from the data analyses.

\section{Behavioral consequences}

\section{Aversive behaviors}

Mean total aversive scores to the infusion of either distilled water or quinine are shown in Figure $2 A$. A two-factor ANOVA indicated significant main effects of stimulus $\left(F_{(1,65)}=92.55 ; p<0.001\right)$ and nerve condition $\left(F_{(6,65)}=7.26 ; p<0.001\right)$, as well as a significant interaction $\left(F_{(6,65)}=7.713 ; p<0.001\right)$. When water was the stimulus, few, if any, aversive behaviors were elicited regardless of the condition of the GL. On the contrary, infusion of quinine elicited many aversive behaviors, but only in animals with intact nerves, i.e., SHAM and REG groups. Comparisons between these nerve-intact groups established that rats with recovered CV taste bud fields were behaviorally indistinguishable from controls regarding total aversive responses to quinine infusion. On the other hand, for rats with a compromised CV field (GLX and PRE groups), quinine-elicited aversive scores were low and comparable with those obtained when water was the stimulus (Fig. $2 A$ ).

The gape was the quinine-elicited aversive behavior elicited most frequently in SHAM rats, representing $\sim 70 \%$ of the total aversive score to this stimulus (Fig. 1B). An intriguing and unanticipated finding was that the number of gapes in quinine-stimulated SHAM rats (both 17 and $94 \mathrm{~d}$ groups combined) was highly correlated $(r=$ $0.81 ; p<0.02$ ) with the number of $\mathrm{CV}$ taste buds (Fig. $1 C$ ). A two-factor ANOVA of gapes indicated significant main effects of stimulus $\left(F_{(1,65)}=80.72 ; p<0.005\right)$ and nerve condition $\left(F_{(6,65)}=\right.$ $5.44 ; p<0.005)$, as well as a significant interaction $\left(F_{(6,65)}=7.713\right.$; $p<0.005$ ). As has been reported previously (Travers et al., 1987; Grill et al., 1992), gapes were significantly attenuated after bilateral transection of the GL $(p<0.005)$. Regeneration after $52 \mathrm{~d}$ led to the re-establishment of quinine-elicited gapes that were comparable in number with control values. PRE-52 rats behaved in a manner similar to that of GLX rats. That is, quinine-elicited gapes were not re-established; rather, the number of gapes to quinine was minimal and equivalent to the number of gapes elicited by water. In a similar manner, regeneration for $94 \mathrm{~d}$ restored the number of quinine-elicited gapes to control values, but $94 \mathrm{~d}$ were not sufficient to generate the normal number of gapes if the nerves had not recovered.

Chin rubs, comprising $22 \%$ of the aversive behaviors in SHAM rats, also were affected by GL nerve section, regeneration, and its prevention in a manner consistent with the effects reported for gapes (Table 2). A two-factor ANOVA of chin rubs indicated significant main effects of stimulus $\left(F_{(1,65)}=32.36 ; p<0.001\right)$ and nerve condition $\left(F_{(6,65)}=4.00 ; p=0.002\right)$, as well as a significant interaction $\left(F_{(6,65)} \stackrel{=}{=} 4.00 ; p=0.002\right)$. Intact nerves, whether unoperated or regenerated, supported more occurrences of this quinine-elicited behavior compared with nonregenerated nerves (all $p$ values $<0.01$ ). There were few HSs and FFs (Table 2), precluding a meaningful statistical analysis.

\section{Ingestive behaviors}

Mean total ingestive scores associated with the inf usion of water or quinine are shown in Figure $2 B$. A two-factor ANOVA indicated no main effects of stimulus $\left(F_{(1,65)}=3.44 ; p=0.068\right)$ or nerve condition $\left(F_{(6,65)}=1.53 ; p=0.181\right)$, nor was there a significant interaction $\left(F_{(6,65)}=2.12 ; p=0.063\right)$. Although there were no significant main effects, it interesting to note that nerve transection (GLX and PRE groups) tended to increase the occurrence of ingestive behaviors elicited by quinine (Fig. $2 B$ ).

\section{Central consequences}

\section{FLI in gNST subfields}

Similar to our previous findings (King et al., 1999), quinine and water evoked similar numbers of FLI neurons only within the nongustatory portion of the NST in SHAM-17 rats $(41.67 \pm 11.92$ and $47.50 \pm 7.24$, respectively). No experimental manipulation produced a significant difference from these values. Within the gustatory portion of the nucleus (sum of the four levels anterior to area postrema), however, a four-way mixed ANOVA indicated a significant main effect of stimulus $\left(F_{(1,65)}=43.11 ; p<0.001\right)$, nerve condition $\left(F_{(6,65)}=2.79 ; p=0.018\right)$, rostrocaudal gNST level $\left(F_{(3,195)}=78.61 ; p<0.001\right)$, and gNST subfield $\left(F_{(5,325)}=72.21\right.$; $p<0.001)$. There were numerous significant two-way, three-way, and four-way interactions. Accordingly, we conducted more simple ANOVAs, followed by post hoc tests where appropriate, to discern the basis for the significant effects.

Mean total numbers of FLI neurons $\left(F_{(13,65)}=6.02 ; p<0.005\right)$ were very different depending on the stimulus and on the integrity 
Figure 3. Summed mean + SE total number of FLI neurons for each subfield pooled across the four standardized rostrocaudal levels of gNST. In $A$, a schematic diagram of these subfields in a coronal section of gNST (medial is to the right) is provided. In subfield $1(A)\left(F_{(13,65)}=2.14 ; p<0.02\right)$, subfield $2(B)\left(F_{(13,65)}=2.94 ; p<0.005\right)$, and subfield $3(C)$ $\left(F_{(13,65)} \stackrel{(13,65)}{=} 2.03 ; p<0.03\right)$, modest quininestimulated FLI activity was observed in SHAM rats that was comparable with water-stimulated subjects. In general, the surgical manipulations did not result in a consistent pattern of effects within these three subfields. In subfield $4(D)\left(F_{(13,65)}=3.91 ; p<\right.$ $0.005)$, subfield $5(E)\left(F_{(13,65)}=13.66 ; p<0.005\right)$ and subfield $6(F)\left(F_{(13,65)}=8.98 ; p<0.005\right)$, quinine-stimulated FLI values far exceeded waterstimulated values in SHAM rats, especially within subfield 5. Bilateral GL nerve transection selectively attenuated quinine-elicited FLI in subfields 4, 5, and 6 . When the GL regenerated, numbers of FLI neurons were restored to control values in as few as 52 d. If, however, the transection was permanent, quinine-stimulated FLI was no greater than that obtained when water was the stimulus. Caret and double caret represent a significant difference from the quinine-stimulated PRE-52 and PRE-94 groups, respectively.
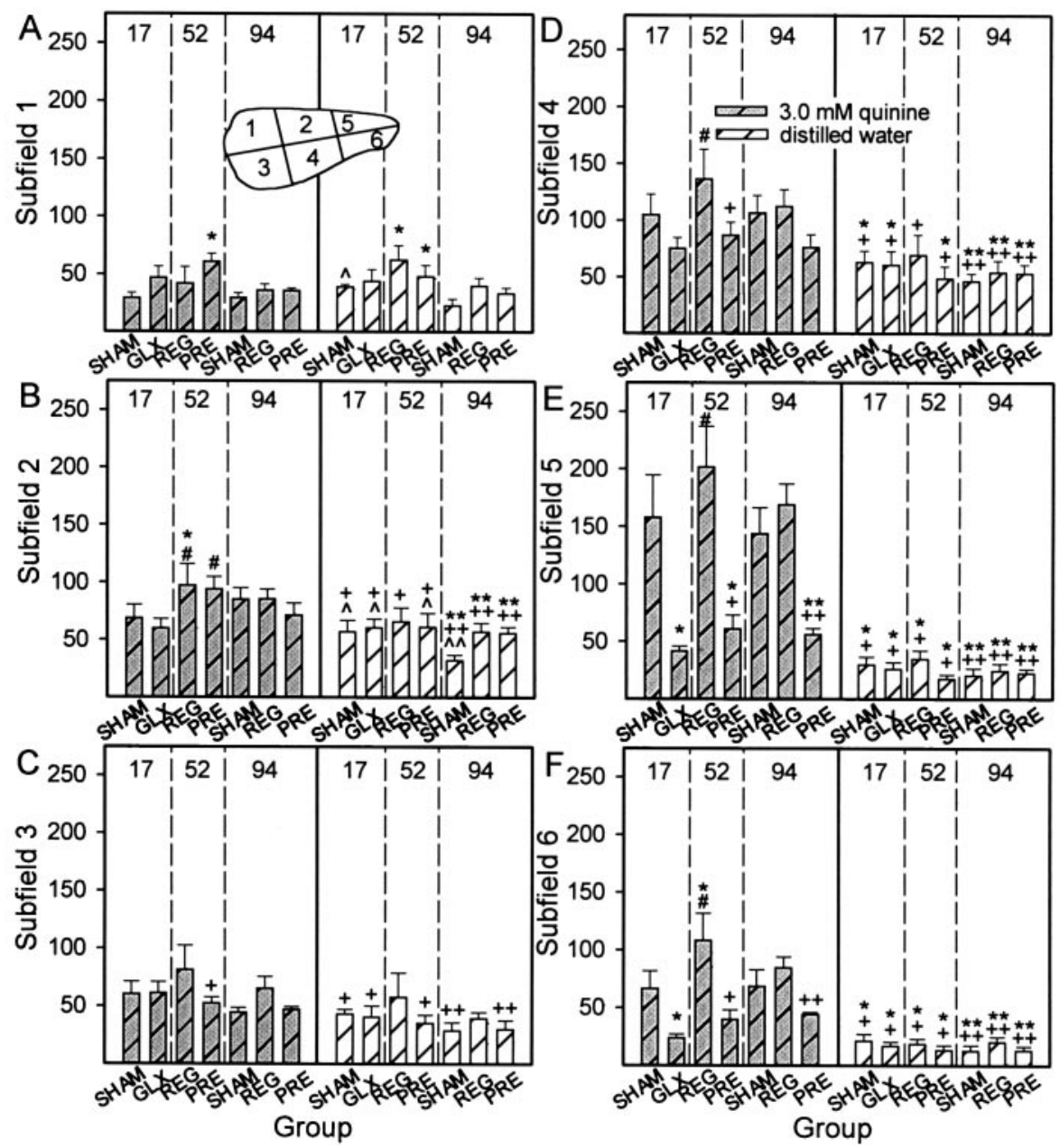

of the nerve (see below). The numbers of quinine-elicited FLI neurons in SHAM-17 (483.83 \pm 85.99) and SHAM-94 (490.97 \pm 75.04) rats were significantly greater than water-elicited mean numbers of FLI neurons $(255.33 \pm 21.97$ and $164.33 \pm 31.44$, respectively; all $p$ values $<0.01$ ). Interestingly, the mean numbers of FLI neurons found in the SHAM-17 rats in the current study were not statistically different (two-sample $t$ tests; all $p$ values $>0.39$ ) from the means we reported previously for similarly stimulated SHAM-17 groups (King et al., 1999). In that study, we observed $615.66 \pm 120.83$ quinine-stimulated FLI neurons and $279.00 \pm$ 36.48 water-stimulated FLI neurons in sham-operated subjects.

When FLI neurons across the rostrocaudal extent of the gNST were summed for each subfield (Fig. 3), several findings from our previous report were confirmed. These include the following: (1) in the SHAM-17 group, quinine did not elicit more FLI than water in subfields 1,2 , or 3; (2) in subfields 4, 5, and 6, quinine-elicited FLI neurons were significantly more numerous than water-stimulated FLI neurons; and (3) this population of quinine-stimulated FLI neurons was significantly attenuated by GL transection. We extend those findings by demonstrating that, with regeneration of GL for either 52 or $94 \mathrm{~d}$, the number of FLI neurons was similar to SHAM values within each of subfields 4,5 , and 6 , whereas the prevention of GL regeneration yielded numbers comparable with quininestimulated GLX rats and water-stimulated controls.

These effects were not restricted to any one level of the gNST. In Figure 4, FLI for subfield 5 at each rostrocaudal level of the gNST is presented because it is the subfield showing the greatest FLI after quinine stimulation. Notice the following at each level: (1) water stimulation elicited little FLI activity compared with quinine stimulation in SHAM rats; (2) GLX attenuated the number of quinine-stimulated FLI neurons; (3) regeneration of the GL restored then numbers of FLI-positive neurons to control values, resulting in significant increases (all $p$ values $<0.01$ ) in the REG-52 group at the RgNST and ICgNST levels; and last, (4) prevention of regeneration, for either 52 or $94 \mathrm{~d}$, resulted in fewer quininestimulated FLI neurons compared with controls (all $p$ values $<$ $0.001)$ with one exception. In RgNST, the numbers of quininestimulated FLI neurons in both PRE groups were not statistically different from their control values. Comparisons between the PRE and REG groups, however, did yield statistical differences (all $p$ values $<0.01$ ), with the numbers of quinine-stimulated FLI neurons being greater in the REG groups. Apparently, $52 \mathrm{~d}$ of nerve regeneration were sufficient to re-establish the number of quininestimulated FLI neurons in the gNST. On the other hand, as many as $94 \mathrm{~d}$ were not enough to produce recovery of neuronal activity if the nerves had not regenerated.

\section{Distribution of FLI neurons}

Irrespective of the numbers of FLI neurons in each group, the relative spatial distributions of these labeled cells across the subfields of the gNST were calculated to compare patterns of distribution. The mean numbers of FLI neurons across the six subfields were compared between groups using Pearson's $\chi^{2}$ test. For this analysis, the quinine-stimulated 17 and 96 d SHAM groups were combined, as were the respective water-stimulated SHAM groups. The 52 and $94 \mathrm{~d}$ REG groups were also combined, and the GLX and PRE groups were collapsed as well. In quinine-stimulated SHAM rats (Fig. 5A), the distribution of FLI neurons within the gNST was clearly distinguishable from the pattern obtained when water (Fig. $5 C$ ) was the stimulus $\left(\chi^{2}=48.7 ; p<0.001\right)$. In contrast, the concordance between the distribution of quinine-stimulated FLI neurons in GLX and PRE rats (Fig. 5D) and that obtained in water-stimulated SHAM controls was striking, and these patterns did not significantly differ $\left(\chi^{2}=2.21 ; p=0.82\right)$. The pattern of 


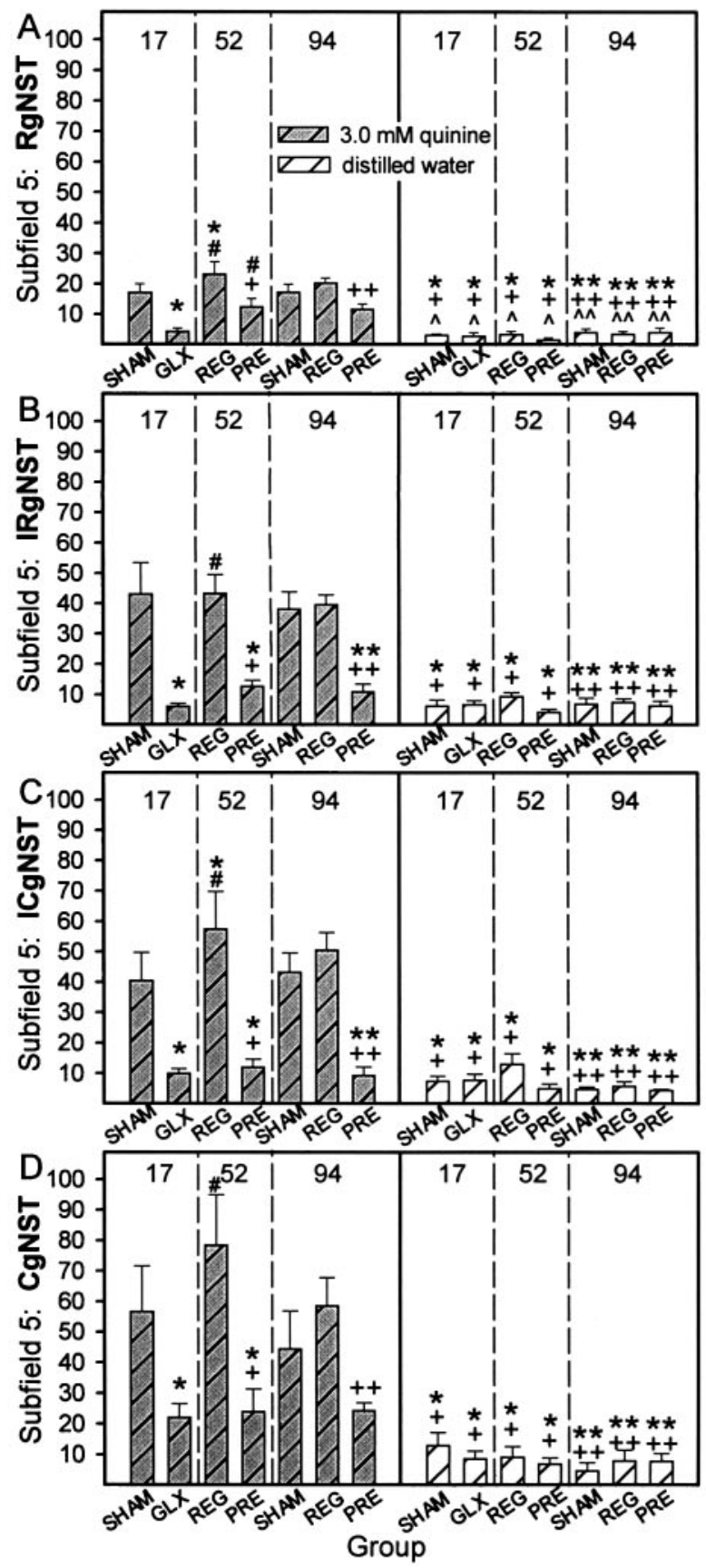

Figure 4. Mean \pm SE number of FLI neurons observed at each rostrocaudal level of the gNST for subfield 5. Note that, for each level, $\operatorname{RgNST}(A)$ $\left(F_{(13,65)}=14.52 ; p<0.005\right), \operatorname{IRgNST}(B)\left(F_{(13,65)}=14.56 ; p<0.005\right)$ $\operatorname{ICgNST}(C)\left(F_{(13,65)}=13.15 ; p<0.005\right)$, and $\operatorname{CgNST}(D)\left(F_{(13,65)}=7.65\right.$; $p<0.005)$, a similar pattern of effects was maintained. Subjects with injured and nonrecovered GL nerves (GLX and PRE groups) exhibited quininerelated FLI activity comparable with water-stimulated rats, whereas the subjects with intact GLs (SHAM and REG groups) showed comparable numbers of quinine-stimulated FLI that were significantly greater than water-stimulated FLI values. Interestingly, within $\operatorname{RgNST}(A)$ and $\operatorname{ICgNST}$ $(C)$, significantly more quinine-stimulated FLI was observed in the REG-52 group than in the SHAM-17 quinine-stimulated group.

quinine-stimulated FLI in the GLX and PRE rats did, however, significantly differ from that seen in the SHAM and REG groups (all $\chi^{2}>47.1 ; p<0.001$ ). In REG rats, the distribution of quinine-stimulated FLI neurons (Fig. $5 B$ ) was remarkably similar to the quinine-stimulated SHAM pattern $\left(\chi^{2}=1.74 ; p=0.88\right)$. The representative photomicrographs in Figure 6 clearly demonstrate the overall effects of nerve transection, regeneration, and the prevention of regeneration on quinine-elicited FLI in the gNST.

\section{FLI in gNST subdivisions}

When the gNST was delineated on the basis of its classic subdivisions and the FLI neurons from these subdivisions were summed for the three most rostral levels of the gNST in SHAM-17 and SHAM-94 rats, the only subdivision in which quinine-stimulated FLI (181.50 \pm 42.15 and $193.29 \pm 27.40$, respectively) exceeded water-stimulated FLI $(61.83 \pm 11.36$ and $39.00 \pm 7.65$, respectively) was the $\mathrm{RC}$ (all $p$ values $<0.001$ ), confirming previous reports (Harrer and Travers, 1996; King et al., 1999). Transection of the GL selectively attenuated the quinine-stimulated population of FLI neurons $(65.17 \pm 9.95 ; p<0.001)$ because no decreases were found in the RL, V, or $M$ subdivisions. Regeneration for either 52 or $94 \mathrm{~d}$ resulted in the appearance of numerous FLI neurons in RC (247.33 \pm 45.63 and $215.33 \pm 19.93$, respectively). Indeed, in REG-52 rats, the number of FLI neurons was not comparable but was significantly greater than that observed in the $\mathrm{RC}$ of SHAM-17 rats $(p<0.001)$. When regeneration was discouraged (PRE groups), the numbers of FLI neurons in the RC (PRE-52, $65.00 \pm 8.69$; PRE-94, $87.00 \pm 24.58)$ were comparable with those found in GLX rats. Importantly, these quininestimulated FLI values were not significantly different from waterstimulated control values (SHAM-17, $61.83 \pm 11.36$; SHAM-94, $39.00 \pm 7.65)$.

\section{DISCUSSION}

\section{Recovery of both quinine-elicited gaping and topographic pattern of FLI in the gNST in GLX rats requires nerve regeneration}

Quinine is a very effective oral stimulus for eliciting oromotor rejection responses, most notably the gape, in rats. Intraoral infusion of this alkaloid stimulus, reported as "bitter-tasting" by humans, induces a characteristic pattern of neuronal FLI in the gNST with the core of activation positioned in the dorsomedial portion of the nucleus (primarily in the medial extent of RC or subfield 5), an area that is considered to be a primary source of neurons projecting to the parabrachial nuclei (Halsell et al., 1996). Bilateral transection of the GL, producing sensory denervation of the posterior tongue, markedly attenuates quinine-induced gaping and changes the magnitude and pattern of FLI in the gNST in response to intraoral quinine delivery such that it resembles what is seen after water stimulation. All of the above findings have been reported previously and are clearly evident in this experiment (Travers et al., 1987; Grill et al., 1992; Harrer and Travers, 1996; King et al., 1999).

In this study, we extend those findings by showing that, when the GL regenerates, both quinine-induced oromotor behavior and FLI are indistinguishable from that observed in sham-operated control rats. The number of gapes, the number of Fos-labeled neurons, and their spatial distribution did not differ between quinine-stimulated sham-operated and nerve-regenerated groups. These findings strongly suggest that the gNST maintains its potential to restore accurately the organization of neural activity that is disrupted by neurotomy, provided the nerve regenerates.

A parallel can be found in recent reports examining the vibrissal sensory system of the rat (Kis et al., 1999). Within a large area of the brainstem trigeminal nucleus (the first synaptic level in the system), no physiological responses were evoked by ipsilateral vibrissal deflection immediately after transection or crush of the infraorbital nerve (ION), which innervates the whiskers. Within 22-35 d, stimulus-evoked responses reappeared in nerve-transected rats. On the contrary, only 7-9 $d$ were required for the reappearance of responses in nerve-crushed subjects, suggesting that the response recovery was related to the integrity of the ION nerve fibers (Kis et al., 1999). With permanent transection of the ION (regeneration being prevented), Waite (1984) reported that the majority of deafferented cells within the trigeminal nucleus remained unresponsive as long as $60 \mathrm{~d}$ after transection. Likewise, in the current study, if the GL did not regenerate, there was no indication that quinine-elicited FLI activity began to return to its normal pattern at this brainstem level. Evidently, the specific neu- 
Figure 5. Mean \pm SE proportion of FLI neurons in each subfield for different stimulus and nerve status conditions. Quinine-stimulated $(A)$ and waterstimulated SHAM $(C)$ rats (both 17 and 94 d groups combined) elicited distinctly different patterns of FLI distribution across the mediolateral plane of the gNST. Notably, the profile of quinine-stimulated FLI $(B)$ in REG groups (both 52 and $94 \mathrm{~d}$ groups combined) mirrored that found for the quininestimulated SHAM subjects (compare $A, B$ ). $D$, In GLX and PRE groups (all combined), the FLI pattern obtained after quinine stimulation was similar to that obtained with water-stimulated SHAM rats (compare $C, D$ ), suggesting that the "neural representation" of quinine was indistinct from water in rats with nonrecovered GL nerves. $Q$, Quinine; $W$, water.

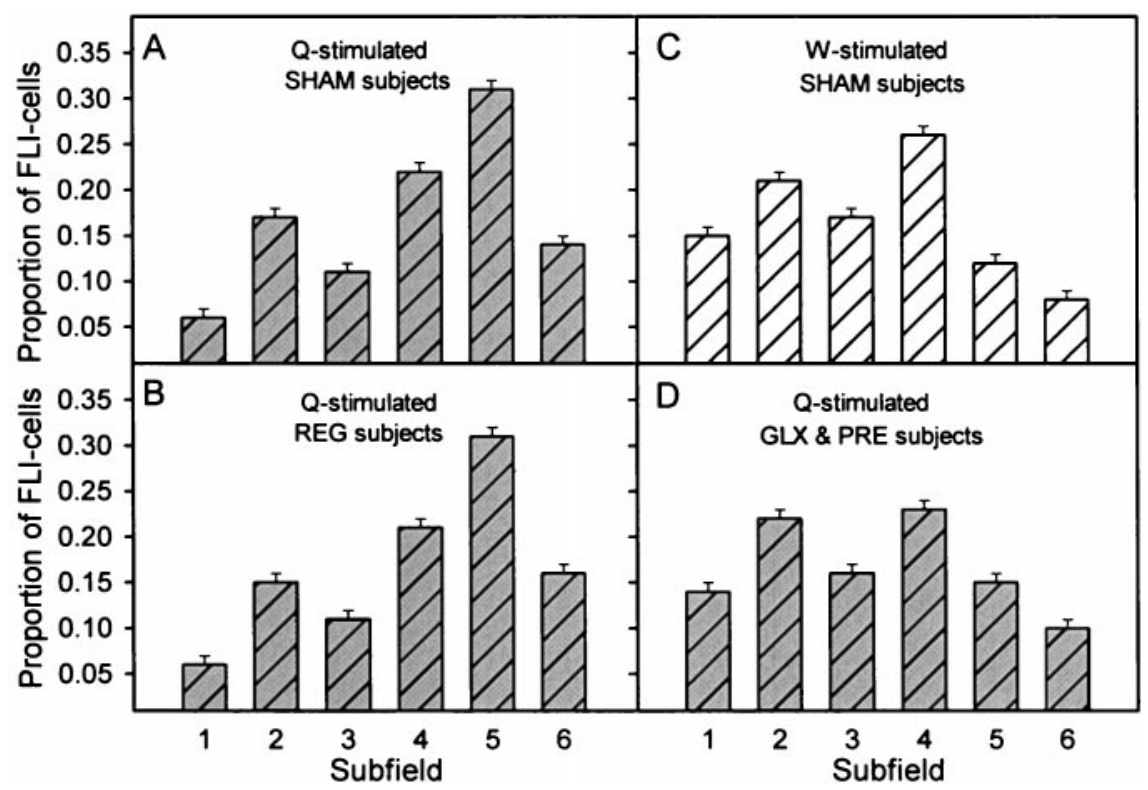

Figure 6. Photomicrographs of FLI in coronal sections taken from the ICgNST level from a quinine-stimulated SHAM-94 rat $(A)$, a quinine-stimulated REG-94 rat $(B)$, a water-stimulated SHAM-17 rat $(C)$, and a quinine-stimulated PRE-52 rat $(D)$. Note (1) the similarity between the quinine-stimulated FLI in the SHAM and REG subjects and (2) the similarity between the quinine-stimulated FLI in the PRE subject and the water-stimulated SHAM subject. $t$, Solitary tract. Scale bar, $100 \mu \mathrm{m}$.
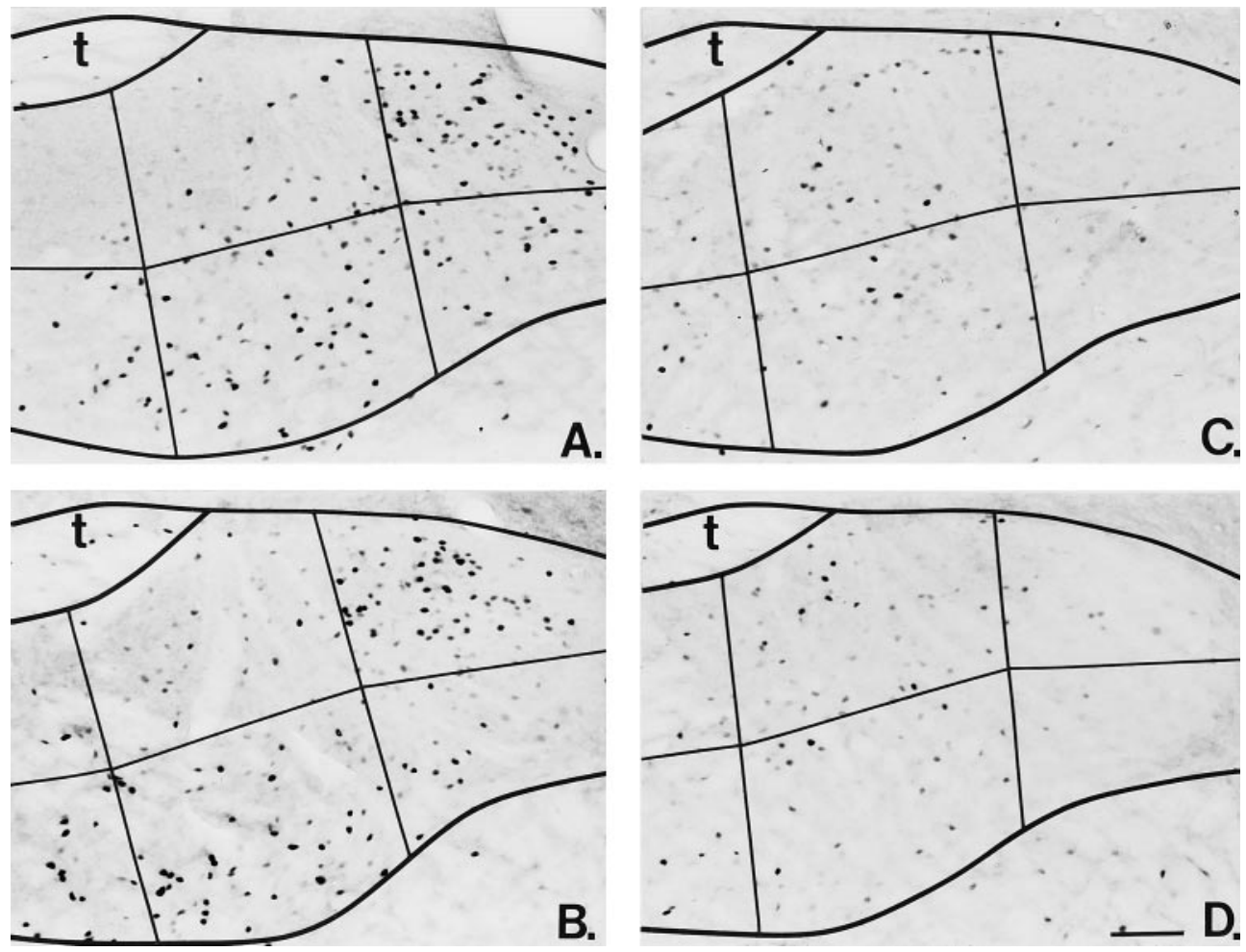

ral (and behavioral) consequences of "permanent" GL transection are long-lasting. The fact, however, that functional recovery was not evident over time alone (i.e., in the PRE groups), at least as assessed by quinine-elicited oromotor behavior and FLI at a primary level of the gustatory neuroaxis, does not preclude possible changes, Fos-related or otherwise, occurring downstream along the central gustatory pathway.

Clearly, the recovery of quinine-elicited aversive consummatory responses in GL-transected rats requires regeneration of the nerve, a finding that is consistent with those from CT-transected rats trained and tested on salt discrimination tasks. Specifically, CT transection in rats raises the $\mathrm{NaCl}$ detection threshold by one to two orders of magnitude (Spector et al., 1990; Slotnick et al., 1991) and substantially impairs performance on a $\mathrm{NaCl}$ versus $\mathrm{KCl}$ taste discrimination task (Spector and Grill, 1992). These particular sensory impairments are entirely reversed by regeneration of the
CT nerve (St. John et al., 1995; Kopka and Spector, 1999; Kopka et al., 2000). It has also been reported that the return of $\mathrm{NaCl}$ taste recognition and c-Fos staining by $\mathrm{CT}$-damaged hamsters relies on nerve regeneration (Barry and Larson, 1993; Barry et al., 1993).

\section{Correlations among CV taste bud number, quinine- induced FLI in the gNST, and quinine-elicited gapes}

One of the noteworthy findings of this study was that the number of gapes elicited by quinine correlated very well with the number of taste buds in the $\mathrm{CV}$ in sham-operated rats $(r=0.81)$. This relationship contrasts with the failure to find significant relationships between the number of anterior tongue taste buds and salt detection and discrimination performance in intact rats (St. John et al., 1995; Kopka and Spector, 1999; Kopka et al., 2000). Nevertheless, the correlation between gapes and $\mathrm{CV}$ taste bud number 
strengthens the link between the input of the GL and oromotor rejection behavior.

Although it was clear that the maintenance of a normal quinineinduced pattern of FLI in the gNST depended on an intact GL, there was no relationship evident between the numbers of FLI neurons and numbers of CV taste buds. Assuming that the strength of the peripheral signal is a critical feature that determines the magnitude of FLI, the failure to find a relationship between CV taste bud number and FLI in the gNST is puzzling. However, based on the anatomical positions of the central terminations of the gustatory nerves compared with the distribution of quinineinduced Fos expression, it is likely that many of the quininestimulated FLI neurons were not second-order neurons receiving direct peripheral input from GL. Some quinine-stimulated FLI neurons were found in areas of the gNST in which only diffuse, if any, terminations from the GL have been reported (e.g., rostrally and ventrally).

These observations also lead to the speculation that the number of CV taste buds is not necessarily an indication of the strength of the GL signal and that the basis of the correlation between taste buds and gapes lies elsewhere. Although there was no relationship between the magnitude of the FLI response in the gNST and gapes observed in our study, DiNardo and Travers (1997) showed a striking correlation $(r=0.90)$ between the number of quinineelicited gapes and the number of FLI neurons overall in the medullary reticular formation (RF). However, for at least one of the lateral (sensory) subdivisions of the medullary RF, the correlation between gapes and FLI was nonsignificant, although it contained overall more FLI neurons compared with the medial (motor) subdivisions for which the correlations were significantly high. The authors suggested that the degree of FLI expression in a particular subdivision of the RF, after the occurrence of quininestimulated oromotor activity, depended on how direct the access of the activated neurons was to the oromotor nuclei. It follows, then, that quinine-induced FLI in the gNST, which sends some projections to the lateral medullary RF (Norgren, 1978) (for review, see Travers, 1993; Halsell et al., 1996) and thus is further removed from the oromotor nuclei, would not necessarily be correlated with oromotor behavior. Nevertheless, it remains to be seen whether numbers of FLI neurons at other levels of the gustatory system might be more strongly correlated with the occurrence of quinineelicited gapes.

\section{The quinine-induced FLI pattern in the gNST and gapes return to normal despite regeneration of only three- fourths of the typical complement of CV taste buds}

Even after $94 \mathrm{~d}$, the numbers of regenerated CV taste buds were approximately three-fourths of that observed in sham-operated control rats. It is noteworthy that this significantly reduced population of posterior tongue taste buds was sufficient to re-establish normal oromotor rejection behaviors (Fig. $2 A$ ), especially gapes (Fig. $1 B$ ), as well as the characteristic pattern of FLI in the gNST (Figs. 3-6). These findings parallel reports examining behavioral proficiency related to $\mathrm{CT}$ regeneration in rats in which performance in salt detection and discrimination tasks was normal despite the reappearance of only $\sim 70 \%$ of the anterior tongue taste buds (St. John et al., 1995; Kopka and Spector, 1999; Kopka et al., 2000).

One explanation for the sufficiency of the reduced number of taste buds to support normal levels of both FLI in the gNST and oromotor rejection behavior is that some type of neural compensatory event occurred. The possibility that some process in regenerated taste bud fields compensates for the reduced number of taste buds to maintain a normal signal from the periphery, although speculative, receives some indirect support from electrophysiological findings in the literature. Cain et al. (1996) reported that relative whole-nerve responses to taste stimuli were normal by $4-8$ weeks after CT nerve crush in hamsters. Additionally, single-fiber responses of the regenerated $\mathrm{CT}$ in gerbils have been shown to be similar to those of the undamaged CT (Cheal and Oakley, 1977;
Cheal et al., 1977). Ninomiya (1998) demonstrated that the distribution of fiber types, based on sensitivity to amiloride, was not substantially different between the intact and the regenerated CT or GL in mice. Moreover, the mean relative spike rates in response to taste stimuli tested in the regeneration group were not different from those in intact control mice.

Such peripheral compensation could perhaps be related to an increase in the number of taste receptor cells per taste bud or an increase in the density of relevant molecular receptor sites in the apical membrane. Now that a family of genes encoding mammalian taste receptors that bind with bitter-tasting compounds has been discovered, the possibility that there are changes in posterior tongue taste receptor function of GL-regenerated rats can come under more direct scrutiny (Adler et al., 2000; Chandrashekar et al., 2000; Matsunami et al., 2000). It should be noted, however, that evidence for such potential peripheral compensatory events is not necessarily ubiquitous across mammalian species. For example, in the cat, electrophysiological activity in the CT was severely depressed at least up to 12 weeks after its transection (Robinson, 1989).

It is also possible that normal behavior and the FLI response depend only on the presence of a critical number of posterior tongue taste buds. Three PRE-94 subjects receiving quinine were eliminated from formal data analysis because they showed $\sim 50 \%$ of the ordinary number of CV taste buds. Nevertheless, the magnitude of both their oromotor rejection behavior and their FLI activity in response to quinine stimulation were well within the average range. In one of these subjects, the GL regenerated unilaterally and a normal pattern of quinine-induced FLI was observed on this regenerated side. The contralateral side displayed the water-like pattern of Fos-labeled neurons, and yet, the rat was behaviorally competent in response to quinine stimulation. Collectively, the data from these discarded subjects suggests that even half of the normal complement of $\mathrm{CV}$ taste buds is adequate to support characteristic patterns of neuronal activity and oromotor rejection behaviors to quinine.

\section{Final remarks}

The Fos technique has been a useful tool in advancing our understanding of gustatory system function. It, however, possesses certain limitations (King et al., 1999), including foremost the likelihood that the observed quinine-induced FLI may represent only a subpopulation of all the neurons activated by the taste stimulus. Caveats notwithstanding, it is still instructive to ask how the manipulation of gustatory input affects the distribution of that subpopulation within the gNST. Indeed, the present investigation has demonstrated that, when GL input is permanently removed, the system is incapable of supporting oromotor rejection behavior and normal patterns of neuronal activity stimulated by oral quinine delivery. There were long-lasting and parallel decreases in the mean numbers of posterior tongue taste buds, gapes, and FLI neurons in the quinine-stimulated GL-transected rats that did not display regeneration (Figs. $1 B, C, 3 E$ ). In contrast, regeneration of the GL led to the recovery of quinine-elicited oromotor behavior and the characteristic neuronal pattern of FLI in the gNST, although CV taste bud numbers were only three-fourths of their typical amount. The underlying basis of this effect may relate to peripheral and/or central changes. It will be important for future studies to define the functional boundaries of the behavioral and neural consequences of the loss and return of gustatory nerve input.

\section{REFERENCES}

Adler E, Hoon MA, Mueller KL, Chandrashekar J, Ryba NJP, Zuker CS (2000) A novel family of mammalian taste receptors. Cell 100:693-702. Barry MA (1999) Recovery of functional response in the nucleus of the solitary tract after peripheral gustatory nerve crush and regeneration. J Neurophysiol 82:237-247.

Barry MA, Larson DC (1993) Recovery of taste-induced expression of $\mathrm{c}$-fos in the CNS after peripheral nerve crush and regeneration. Soc Neurosci Abstr 19:678.

Barry MA, Larson DC, Frank ME (1993) Loss and recovery of sodium- 
salt taste following bilateral chorda tympani nerve crush. Physiol Behav 53:75-80.

Cain P, Frank ME, Barry MA (1996) Recovery of chorda tympani nerve function following injury. Exp Neurol 141:337-346.

Chandrashekar J, Mueller KL, Hoon MA, Adler E, Feng L, Guo W, Zuker CS, Ryba NJP (2000) T2Rs function as bitter taste receptors. Cell 100:703-111.

Cheal M, Oakley B (1977) Regeneration of fungiform taste buds: temporal and spatial characteristics. J Comp Neurol 172:609-626.

Cheal M, Dickey WP, Jones LB, Oakley B (1977) Taste fiber responses during reinnervation of fungiform papillae. J Comp Neurol 172:609-626.

DiNardo LA, Travers JB (1997) Distribution of Fos-like immunoreactivity in the medullary reticular formation of the rat after gustatory elicited ingestion and rejection behaviors. J Neurosci 17:3826-3839.

Ganchrow JR, Ganchrow D (1989) Long-term effects of gustatory neurectomy on fungiform papillae in the young rat. Anat Rec 225:224-231.

Grill HJ, Norgren R (1978) The taste reactivity test. I. Mimetic responses to gustatory stimuli in neurologically normal rats. Brain Res 143:263-279.

Grill HJ, Schwartz GJ (1992) The contribution of gustatory nerve input to oral motor behavior and intake-based preference. II. Effects of combined chorda tympani and glossopharyngeal nerve section in the rat. Brain Res 573:105-113.

Grill HJ, Schwartz GJ, Travers JB (1992) The contribution of gustatory nerve input to oral motor behavior and intake-based preference. I. Effects of chorda tympani or glossopharyngeal nerve section in the rat. Brain Res 573:95-104.

Guth L (1957) Effects of glossopharyngeal nerve transection on the circumvallate papilla of the rat. Anat Rec 128:715-731.

Halsell CB, Travers SP, Travers JB (1996) Ascending and descending projections from the rostral nucleus of the solitary tract originate from separate neuronal populations. Neuroscience 72:185-197.

Hamilton RB, Norgren R (1984) Central projections of gustatory nerves in the rat. J Comp Neurol 222:560-577.

Harrer MI, Travers SP (1996) Topographic organization of Fos-like immunoreactivity in the rostral nucleus of the solitary tract evoked by gustatory stimulation with sucrose and quinine. Brain Res 711:125-137.

Jain N, Florence SL, Kaas JH (1998) Reorganization of somatosensory cortex after nerve and spinal cord injury. News Physiol Sci 13:143-149.

King CT, Travers SP, Rowland NE, Garcea M, Spector AC (1999) Glossopharyngeal nerve transection eliminates quinine-stimulated Fos-like immunoreactivity in the nucleus of the solitary tract: implications for a functional topography of gustatory nerve input in rats. J Neurosci 19:3107-3121.

Kis Z, Farkas T, Rabel K, Kis E, Korodi K, Simon L, Marusin I, Rojik I, Toldi J (1999) Comparative study of the neuronal plasticity along the neuroaxis of the vibrissal sensory system of adult rat following unilatera infraorbital nerve damage and subsequent regeneration. Exp Brain Res 126:259-269.

Kopka SL, Spector AC (1999) The effects of chorda tympani transection and regeneration on $\mathrm{NaCl}$ detection threshold. Chem Senses [Abstr] 24:524-525.

Kopka SL, Geran LC, Spector AC (2000) Functional status of the regenerated chorda tympani nerve as assessed in a salt taste discrimination task. Am J Physiol Regul Integr Comp Physiol 278:R720-R731.

Markison S, St. John SJ, Spector AC (1999) Glossopharygeal nerve tran- section reduces quinine avoidance in rats not given presurgical stimulus exposure. Physiol Behav 65:773-778.

Matsunami H, Montmayeur JP, Buck LB (2000) A family of candidate taste receptors in human and mouse. Nature 404:601-604.

Miller IJ (1977) Gustatory receptors of the palate. In: Food intake and chemical senses (Katsuki Y, Sato M, Takagi S, Oomura Y, eds), pp 173-186. Tokyo: Tokyo UP.

Ninomiya Y (1998) Reinnervation of cross regenerated gustatory nerve fibers into amiloride-sensitive and amiloride-insensitive taste receptor cells. Proc Natl Acad Sci USA 95:5347-5350.

Norgren R (1978) Projections from the nucleus of the solitary tract in the rat. Neuroscience 3:207-218.

Robinson PP (1989) The reinnervation of the tongue and salivary glands after lingual nerve injury in cats. Brain Res 483:259-271.

Slotnick BM, Sheelar S, Renatmeister-Bryant H (1991) Transection of the chorda tympani and insertion of ear pins for stereotaxic surgery: equivalent effects on taste sensitivity. Physiol Behav 50:1123-1127.

Spector AC, Grill HJ (1992) Salt taste discrimination after bilateral section of the chorda tympani nerve or the glossopharyngeal nerves. Am J Physiol Regul Integr Comp Physiol 263:R169-R176.

Spector AC, Breslin P, Grill HJ (1988) Taste reactivity as a dependent measure of the rapid formation of conditioned taste aversion: a tool for the neural analysis of taste-visceral associations. Behav Neurosci 102:942-952.

Spector AC, Schwartz GJ, Grill HJ (1990) Chemospecific deficits in taste detection after selective gustatory deafferentation in rats. Am J Physiol Regul Integr Comp Physiol 258:R820-R826.

St. John SJ, Spector AC (1996) Combined glossopharyngeal and chorda tympani nerve transection elevates quinine detection thresholds in rats (Rattus norvegicus). Behav Neurosci 110:1456-1468.

St. John SJ, Spector AC (1998) Behavioral discrimination between quinine and $\mathrm{KCl}$ is dependent upon input from the seventh cranial nerve: implications for the functional roles of the gustatory nerves in rats. J Neurosci 18:4353-4362.

St. John SJ, Garcea M, Spector AC (1994) Combined, but not single gustatory nerve transection substantially alters taste-guided licking behavior to quinine in rats. Behav Neurosci 18:131-140.

St. John SJ, Markison S, Spector AC (1995) Salt discriminability is related to number of regenerated taste buds after chorda tympani nerve transection in rats. Am J Physiol 269:R141-R153.

Travers JB, Grill HJ, Norgren R (1987) The effects of glossopharyngeal and chorda tympani nerve cuts on the ingestion and rejection of sapid stimuli: an electromyographic analysis in the rat. Behav Brain Res 25:233-246.

Travers SP (1993) Orosensory processing in neural systems of the nucleus of the solitary tract. In: Mechanisms of taste transduction (Simon SA, Roper SD, eds), pp 339-394. Boca Raton: CRC.

Waite PM (1984) Rearrangement of neuronal responses in the trigeminal system of the rat following peripheral nerve section. J Physiol (Lond) 352:425-445.

Whitehead MC (1988) Neuronal architecture of the nucleus of the solitary tract in the hamster. J Comp Neurol 276:547-572.

Whitehead MC, McGlathery ST, Manion BG (1995) Transganglionic degeneration in the gustatory system consequent to chorda tympani damage. Exp Neurol 132:239-250. 